\title{
Parametric Optimization of Load Bearing Elements for Composite Wing of an Unmanned Aerial Vehicle
}

\author{
Naing Lin Aung ${ }^{1, *}$, Oleg Tatarnikov ${ }^{1,2}$, and Phyo Wai Aung ${ }^{1}$ \\ ${ }^{1}$ Department of Aerospace Composite Construction, Bauman Moscow State Technical University, \\ Moscow 105005, Russia. \\ ${ }^{2}$ Department of Higher Mathematics, Plekhanov Russian University of Economics, Moscow 117997, \\ Russia.
}

\begin{abstract}
This paper describes the optimizing results of structural elements of the composite wing of an unmanned aerial vehicle. The thickness and composite lay-up structure of load-bearing elements and wing skin are determined using the ANSYS software package. The optimal structure is presented using the Pareto set method of the "ideal center" basing on four criteria: minimum mass, deflection, normal stress, and maximum safety factor of the wing. Verification calculations were carried out to determine the safety factor of the load-bearing wing structure using a geometrically nonlinear model in FEMAP software.
\end{abstract}

\section{Introduction}

An unmanned aerial vehicle (UAV) is defined as a type of aircraft with an engine that does not carry a human pilot, uses aerodynamic force to create a lift, can fly autonomously or remotely, and can carry a payload. In recent decades, the use of UAV has been rapidly expanding in both civilian and military aviation. The weight of the vehicle is always an important parameter, characterizing the technical perfection of the UAV, as this characteristic depends on the maximum altitude and range of flight, as well as the handling of the aircraft and its maneuverability. Currently, most aircraft designs are made of layered composites due to their high specific strength and rigidity characteristics, as well as the relatively high level of technology for composites. This study looked at the two-spars straight wing structure with a wingspan of $10.5 \mathrm{~m}$ and a chord length of $0.78 \mathrm{~m}$. The position of the front and rear spars was fixed at a relative distance of $30 \%$ and $60 \%$ of the chord from the edge of the wing. The distance between the ribs was $400 \mathrm{~mm}$ [1]. The purpose of this work was to determine the optimal design and structural parameters of the composite wing of an unmanned aerial vehicle.

\footnotetext{
*Corresponding author: nainglinaung.cm13@gmail.com
} 


\subsection{Methodology}

The main structural elements of composite wing were considered as the upper and lower skin, two I-beam spars and 15 ribs. Aerodynamic pressure load was distributed on the surface of the lower skin of the UAV wing. As an optimization of the design parameters of the wing were considered in two stages. In the first stage, the thicknesses of the load-bearing elements and wing skin were determined using laminated composite materials: unidirectional CFRP, CFRP fabric and fiberglass. In the second stage, the optimization of the laying up structure of the optimal composite material was considered: CFRP fibric, considering the existing aerodynamic loads. The geometric model of the UAV wing is presented in Fig. 1.

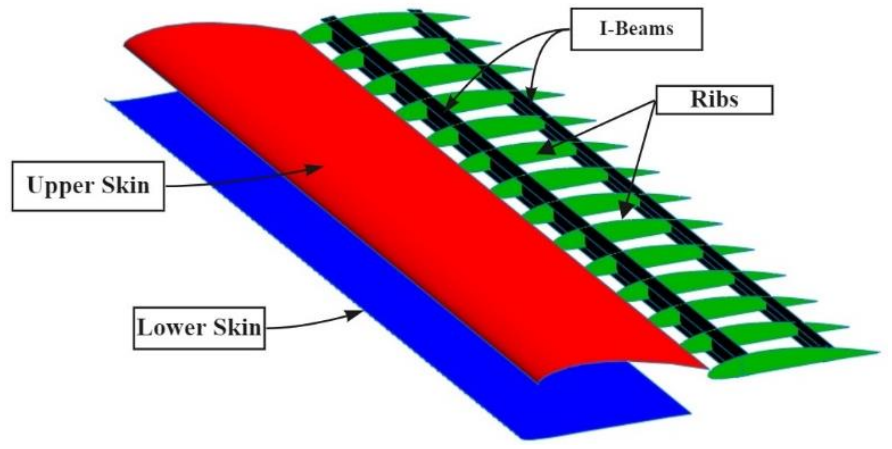

Fig. 1. Two-spar UAV wing model

\subsection{Aerodynamic load acting on the wing.}

The basic technical requirements for the composite UAV wing were accepted in according to the standard of USAR 337. The estimated aerodynamic pressure was determined using the following equations $[2,3]$ :

$$
\begin{gathered}
q_{y}=\frac{G_{0 \cdot g} \cdot n_{\text {max }}^{a} \cdot f \cdot b(z)}{S_{w}}, \\
q_{w}=\frac{G_{w} \cdot g \cdot n_{\text {max }}^{a} \cdot f \cdot b(z)}{S_{\mathrm{w}}}, \\
q_{s}=q_{y}-q_{w}, \\
q_{\Sigma}=\frac{q_{s}}{b(z)} .
\end{gathered}
$$

Where $\mathrm{q}_{\mathrm{y}}$ - aerodynamic load acting on the wing; $\mathrm{q}_{\mathrm{w}}$ - structural load of the wing; $\mathrm{q}_{\mathrm{s}}-$ resulting aerodynamic load of the wing; $\mathrm{q}_{\Sigma}$ - aerodynamic pressure acting on the wing; $G_{0}=$ $450 \mathrm{~kg}$ - UAV take-off mass; $G_{w}=72 \mathrm{~kg}$ - wing mass, $\mathrm{g}$ - acceleration of free fall, $b(z)-$ wing chord length $n^{a}{ }_{\max }=3.8$ - coefficient of load factor; $f=1.5$ - coefficient of safety factor.

\subsection{Material properties}

All the structural elements of UAV wing are made of composite materials layered structure. Three types of materials forming composite layers were considered: unidirectional carbon plastics, woven carbon plastics, and unidirectional E-glass plastic. The mechanical characteristics of these materials are given in the table. 1 [4]. 
Table 1. Mechanical characteristics of composite materials.

\begin{tabular}{|l|l|l|l|}
\hline Properties & $\begin{array}{c}\text { Unidirectional } \\
\text { carbon plastic }\end{array}$ & $\begin{array}{c}\text { woven } \\
\text { carbon }\end{array}$ & $\begin{array}{c}\text { Unidirectional } \\
\text { E-glass }\end{array}$ \\
\hline Longitudinal modulus, GPa & 123.34 & 59.16 & 35 \\
\hline Transverse modulus, GPa & 7.78 & 59.16 & 90 \\
\hline Shear modulus, GPa & 5 & 3.3 & 4.7 \\
\hline Longitudinal tensile strength, MPa & 1632 & 513 & 780 \\
\hline Longitudinal compressive strength, MPa & 704 & 437 & 480 \\
\hline Transverse tensile strength, MPa & 34 & 513 & 31 \\
\hline Transverse compressive strength, MPa & 68 & 437 & 100 \\
\hline Shear strength, MPa & 80 & 120 & 60 \\
\hline Density, kgm ${ }^{-3}$ & 1518 & 1451 & 1850 \\
\hline Thickness layer, mm & 0.15 & 0.15 & 0.2 \\
\hline Poisson's ratio & 0.27 & 0.3 & 0.28 \\
\hline
\end{tabular}

\section{Modeling and simulation calculation}

The optimal thickness of the load-bearing elements was calculated using the OPTISLANG module in the ANSYS software package. The initial values of the thickness of the loadbearing elements and the wing skin were given in the same way for all materials according to Table 2. When choosing the optimal thickness, the wing deflection limit was taken at $5 \%$ of wingspans [5].

Table 2. Thickness optimization parameters for load-bearing elements of UAV wing.

\begin{tabular}{|l|l|c|c|}
\hline \multicolumn{2}{|c|}{ Structural elements } & $\begin{array}{c}\text { Initial thickness } \\
(\mathrm{mm})\end{array}$ & $\begin{array}{c}\text { Variable thickness } \\
(\mathrm{mm})\end{array}$ \\
\hline \multirow{3}{*}{ Wing skin } & Upper & 2 & $1-6$ \\
\cline { 2 - 4 } & Lower & 2 & $1-6$ \\
\hline \multirow{4}{*}{ Front spar } & Upper flange & 2 & $1-6$ \\
\cline { 2 - 4 } & Lower flange & 2 & $1-6$ \\
\cline { 2 - 4 } & Web & 2 & $1-6$ \\
\hline \multirow{3}{*}{ Rear spar } & Upper flange & 2 & $1-6$ \\
\cline { 2 - 4 } & Lower flange & 2 & $1-6$ \\
\cline { 2 - 4 } & Web & 2 & $1-6$ \\
\hline Ribs & 2 & $1-6$ \\
\hline
\end{tabular}

In the first step of optimization of the wing design elements, 400 variants for each material were considered. In the figures $2 \mathrm{a}, 2 \mathrm{~b}$ and $2 \mathrm{c}$ on the plane of the criteria mass and deflection presents Pareto-optimal variants for each material, which are indicated: $i_{g}$ - variant No. $i$ of fiberglass, $\mathrm{j}_{\mathrm{w}}$ - variant No. $\mathrm{j}$ of CFRP fabric, $\mathrm{k}_{\mathrm{u}}$ - variant No $\mathrm{k}$ of unidirectional CFRP. For each material, the optimal variant was chosen using the following Pareto set "ideal center" equation.

$$
L=\sqrt{\frac{\left(d^{i}-d^{i c}\right)}{d_{a v g}^{2}}+\frac{\left(m^{i}-m^{i c}\right)}{m_{a v g}^{2}}}
$$


Where, L - dimensionless distance between $i^{\text {th }}$ variant and ideal center, $d^{i}, m^{i}-$ deflection and mass of $i^{\text {th }}$ variant, $d^{\text {ic }}, m^{\text {ic }}-$ deflection and mass of ideal center, $d_{a v g}$, $\mathrm{m}_{\mathrm{avg}}-$ average deflection and mass value.

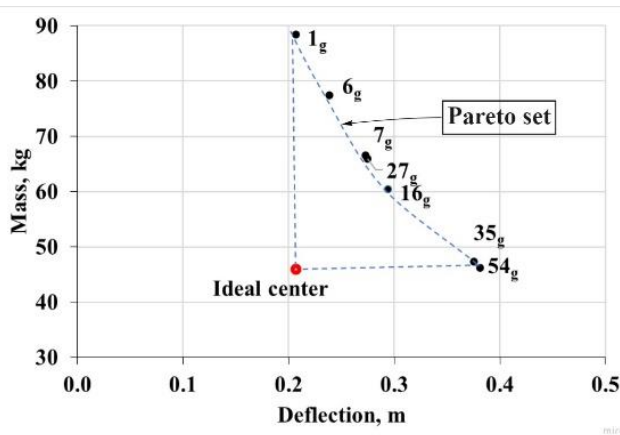

(a)

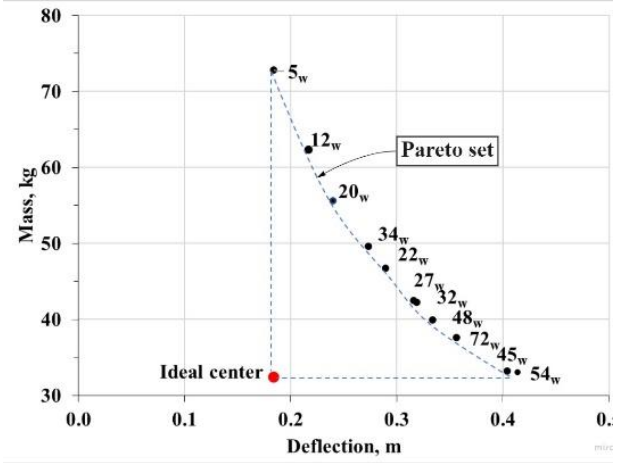

(b)

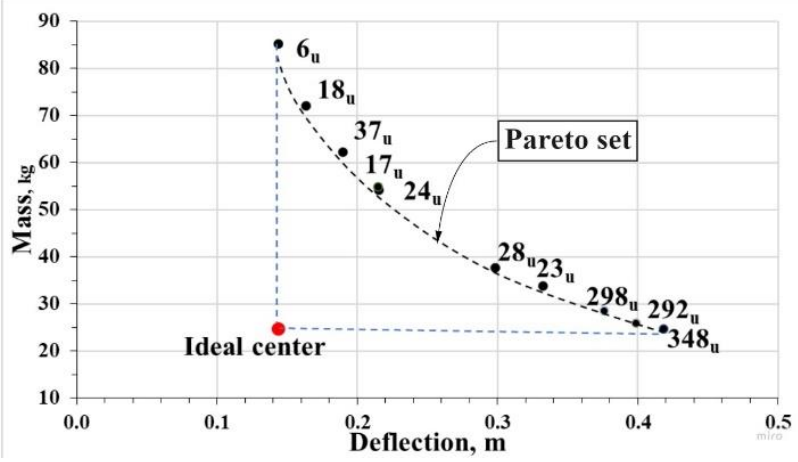

(c)

Fig. 2. Pareto optimal variant: (a) fiberglass (b) CFRP fabric (c) unidirectional CFRP

In the next step, the remaining three variants $6_{\mathrm{g}}, 54_{\mathrm{w}}$ and $28_{\mathrm{u}}$ were determined by the value of the third criteria, margin of safety in the action of aerodynamic pressure. Margin of safety calculations were made using a geometrically non-linear model in the FEMAP software package. Regarding the criteria for minimum mass and maximum margin of safety with using the additional criteria of "ideal center" the best variant was CFRP fabric 54 $4_{\mathrm{w}}$ (Fig. 3).

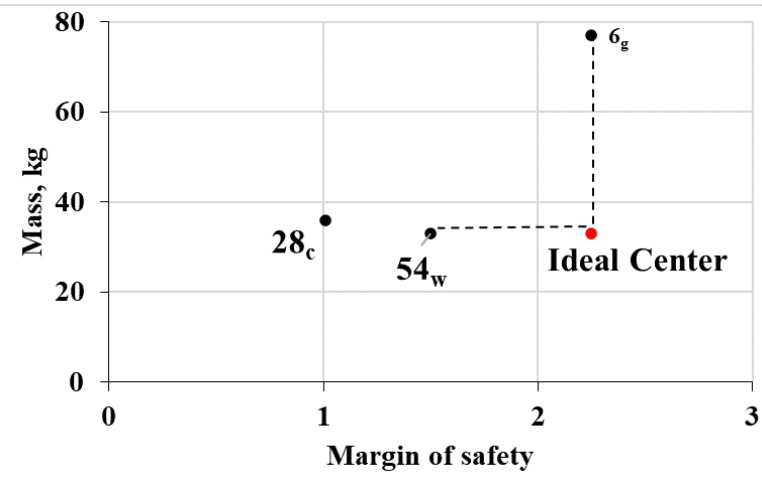

Fig. 3. Optimal variant for three materials 
In table 3 shows the thickness of the structural elements of the composite wing for the optimal variant No. $54_{\mathrm{w}}$.

Table 3. The thickness of load-bearing elements for optimal variant No. $54 \mathrm{w}$.

\begin{tabular}{|l|l|c|}
\hline \multicolumn{2}{|c|}{ Structural elements } & Thickness (mm) \\
\hline \multirow{3}{*}{ Wing skin } & Upper & 2.2 \\
\cline { 2 - 3 } Front spar & Lower & 1.6 \\
\cline { 2 - 3 } & Upper flange & 5.1 \\
\cline { 2 - 3 } & Lower flange & 1 \\
\cline { 2 - 3 } & Web & 2.7 \\
\hline \multirow{3}{*}{ Rear spar } & Upper flange & 1.9 \\
\cline { 2 - 3 } & Lower flange & 2.4 \\
\cline { 2 - 3 } & Web & 1.9 \\
\hline Ribs (No. 1 to 14) & $(1.7,1.4,1.8,1.4,1.9,1.7,1.5,2,1.7,1.1,2,1.6,1,1.3)$ \\
\hline \multicolumn{2}{|l|}{ Mass (kg) } & 32.9 \\
\hline Deflection (mm) & 414.1 \\
\hline
\end{tabular}

\section{Optimization of composite lay-up structure for UAV wing}

The layering angles were optimized using the OPTISLANG module in the ANSYS software complex. The angle of the layers for each constructive wing element ranged from 90 to -90 degrees, with an initial value of 0 degrees [6]. Since at this stage of optimization of the thickness of all the design elements have already been defined and thus the mass of the wing has been fixed, the minimum deflection and minimum normal stress criteria were adopted as optimization criteria. In the process of optimization, 210 variants of the composite lay-up structure were considered. The resulting Pareto-optimal variants are presented in Fig. 4 as points on the criteria plane.

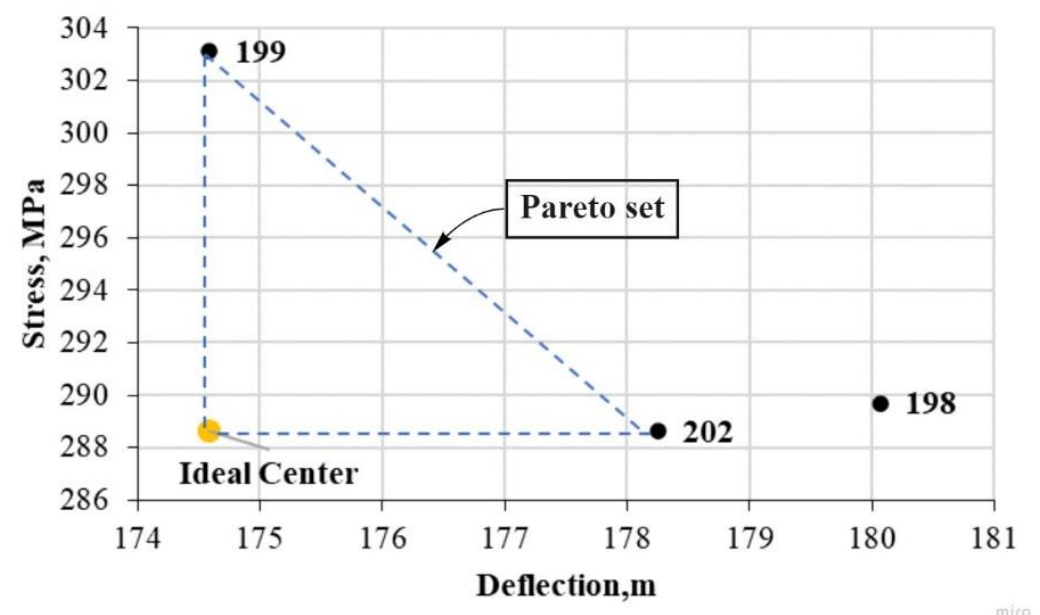

Fig. 4. Pareto-optimal variants for composite lay-up structure

Results of verification calculations by margin of safety of these Pareto-optimal variants showed that only one of them, namely, variant No. 202 meets the specified requirements for the reserve of stability. This variant was chosen as the optimal one for all the criteria considered. The optimal angle of layers for all load-bearing elements and wing skin was obtained as $0, \pm 90$ degrees. 


\section{Conclusions}

1. The optimal thickness of the load bearing elements of the frame and skin of the composite wing is determined by the criteria of minimum mass and deflection for three materials.

2. The optimal material for the load-bearing elements of the composite UAV wing was determined.

3. The optimal lay-up structure of CFRP fabric for load bearing elements of the frame and wing skin have been determined.

4. The strength and stability of the optimized composite wing has been calculated.

\section{References}

1. Naing Lin Aung, Oleg Tatarnikov., Phyo Wai Aung, IOP Conf. Ser. Mater. Sci. Eng. $971052076,(2020)$

2. NATO standartization agreement 4671 (Edition 1 USAR, 2009)

3. Phyo Wai Aung, Oleg Tatarnikov., Naing Lin Aung, IOP Conf. Ser. Mater. Sci. Eng. 971 022058, (2020)

4. E. R Ashikhmina, T. G Ageyeva, P. V Prosuntsov, IOP Conf. Ser. Mater. Sci. Eng. 683 012067, (2019)

5. S V Baranovski, K V Mikhailovskiy, IOP Conf. Ser. Mater. Sci. Eng. 934 012021, (2020)

6. M. Rajadurai, P. Vinayagam, G. Mohana Priya, K. Balakrishnan, 4, 6 (2017) 